\title{
Effects of Repetitive Peripheral Magnetic Stimulation on Patients With Acute Low Back Pain: A Pilot Study
}

\author{
Young-Ho Lim, MD', Ji Min Song, MD¹, Eun-Hi Choi, $\mathrm{MD}^{2}$, Jang Woo Lee, $\mathrm{MD}^{3}$
}

\begin{abstract}
${ }^{1}$ Department of Physical Medicine and Rehabilitation, Hallym University Sacred Heart Hospital, Hallym University College of Medicine, Anyang; ${ }^{2}$ Department of Rehabilitation Medicine, Hallym University Chuncheon Sacred Heart Hospital, Hallym University College of Medicine, Chuncheon; ${ }^{3}$ Department of Physical Medicine and Rehabilitation, National Health Insurance Service Ilsan Hospital, Goyang, Korea
\end{abstract}

Objective To investigate the effects of real repetitive peripheral magnetic stimulation (rPMS) treatment compared to sham rPMS treatment on pain reduction and functional recovery of patients with acute low back pain.

Methods A total of 26 patients with acute low back pain were randomly allocated to the real rPMS group and the sham rPMS group. Subjects were then administered a total of 10 treatment sessions. Visual analogue scale (VAS) was assessed before and after each session. Oswestry Disability Index (ODI) and Roland-Morris Disability Questionnaire (RMDQ) were employed to assess functional recovery at baseline and after sessions 5 and 10.

Results Real rPMS treatment showed significant pain reduction immediately after each session. Sustained and significant pain relief was observed after administering only one session in the real rPMS group. Significant functional improvement was observed in the real rPMS group compared to that in the sham rPMS group after sessions 5 and 10 based on ODI and after session 5 based on RMDQ.

Conclusion Real rPMS treatment has immediate effect on pain reduction and sustained effect on pain relief for patients with acute low back pain compared to sham rPMS.

Keywords Acute low back pain, Repetitive peripheral magnetic stimulation, Pain reduction, Functional recovery

\section{INTRODUCTION}

Low back pain, one of the major causes of disability worldwide, is a very common reason for seeking medi- cal care [1]. Low back pain can be classified based on duration as follows: (1) acute back pain that lasts less than 6 weeks; (2) sub-acute back pain lasting from 6 to 12 weeks; and (3) chronic back pain that persists for more

Department of Rehabilitation Medicine, Hallym University Chuncheon Sacred Heart Hospital, 77 Sakju-ro, Chuncheon 24253, Korea. Tel: +82-33-2405299, Fax: +82-33-255-6244, E-mail: pmnrh@naver.com

ORCID: Young-Ho Lim (https://orcid.org/0000-0002-4446-3854); Ji Min Song (https://orcid.org/0000-0003-0853-0463); Eun-Hi Choi (https://orcid. org/0000-0002-7345-6952); Jang Woo Lee (https://orcid.org/0000-0002-2634-0375).

@ This is an open-access article distributed under the terms of the Creative Commons Attribution Non-Commercial License (http://creativecommons.org/ licenses/by-nc/4.0) which permits unrestricted noncommercial use, distribution, and reproduction in any medium, provided the original work is properly cited. Copyright $\odot 2018$ by Korean Academy of Rehabilitation Medicine 
than 12 weeks. Among these three types, acute low back pain (ALBP) generally demonstrates favorable prognosis with recovery rate ranging from $39 \%$ up to $90 \%$ within 6 weeks [2,3]. However, ALBP patients who seek medical care show an upper margin of moderate level of pain (i.e., average visual analogue scale [VAS] of 6.25) which causes disability (i.e., average Roland-Morris Disability Questionnaire [RMDQ] score of 13.04) [4]. Accordingly, appropriate pain control is required for ALBP patients. As a way to improve the pain and disability that ALBP patients experience, staying active is advised while bed rest is discouraged [5]. However, such advice can be hard to follow when one is in pain. Up to date, options that have shown proven effects for pain reduction with minimal adverse effects in ALBP patients are limited [2,6-10].

Repetitive peripheral magnetic stimulation (rPMS) is a non-invasive treatment method that can penetrate to deeper conductive structure with relatively painless stimulation. Of various studies conducted since 1995, no study has observed any adverse effects with the exception of certain participants reporting tight feelings within a tolearble range. Thus, rPMS can be considered as a treatment method with a high level of tolerance and safety. It has been demonstrated that rPMS has therapeutic effects on musculoskeletal pain [11]. However, no established guideline exists. Thus, further study is required.

Based on literature search, rPMS has been applied to chronic low back pain (CLBP) patients in four studies [1215]. However, no study has observed its effects on ALBP patients. Therefore, the objective of this pilot study was to investigate clinical effects of rPMS on pain reduction and functional recovery in ALBP patients with a randomized controlled design.

\section{MATERIALS AND METHODS}

\section{Subjects}

Patients with ALBP occurring within the last 6 weeks who reported pain with VAS of 3 or higher were recruited. Magnetic resonance imaging study was performed if there was any possibility of a diagnosis other than acute lumbar sprain based on patient's symptoms, physical examination, and a lumber X-ray. Patients with age of 19 years or more who possessed linguistic and cognitive capabilities to explain the degree of pain were selected. Exclusion criteria were: (1) weakness in lower extremities due to lumbar radiculopathy or myelopathy, (2) lumbar fracture, (3) non-mechanical low back pain due to neoplasia, inflammatory arthritis, or infection, (4) pacemaker, (5) history of seizure, pregnancy, malignancy, or (6) history of lumbar surgery. None of participants in this study had any form of magnetic stimulation treatment previously. This study was approved by the Institutional Review Board of Hallym University Chuncheon Sacred Heart Hospital (No. 2017-12).

\section{Methods}

A total of 26 subjects were randomly allocated into real rPMS group and sham rPMS group using a block randomization program. Subjects were blinded to which group they would be allocated into. The purpose of this study was explained to subjects to determine how to perform rPMS treatment more effectively. Patients in the two groups had general physical therapy modality and medication concurrently with the intervention introduced through this study. Neuro MSL magnetic stimulator (MR Inc., Seoul, Korea) was used in this study. It is known that a round coil is more advantageous than a figure- 8 coil for stimulating structures in a deep layer such as spinal roots and covering a larger area such as a paraspinal muscle group [11]. Thus, round coil suitable for treating low back pain was employed in this study. Stimulation site was determined based on patient's most tender point prior to the start of each treatment session. For patients in the real rPMS group, the coil was placed at a flat tangential orientation targeting the most painful lumbar region in prone position. This is the orientation that enables the coil to be positioned parallel to the body surface, thereby maximizing effects of magnetic stimulation applied to the target area. For patients in the sham rPMS group, the coil was applied at a transverse orientation to the most painful lumbar region in prone position. This is the orientation which positions the coil at a $90^{\circ}$ angle to the body surface, thereby minimizing effects of magnetic stimulation applied to the target area [16].

Both groups underwent 10 sessions over a span of 2 weeks. Each session lasted 20 minutes and entailed an intermittent stimulation protocol consisting of 5 seconds of stimulation at a frequency of $20 \mathrm{~Hz}$ followed by 25 seconds of resting. The total number of stimuli over 20 minutes amounted to 4,000 times. The stimulus intensity level for the real rPMS group commenced at $20 \%$ of the 
maximal stimulator output. It was gradually raised by $5 \%$. The final stimulation intensity was determined at the maximum intensity level which induced sufficient contraction of the paraspinal muscle while still falling within tolerable range of the patient $[11,17,18]$. For the sham rPMS group, the stimulus intensity level was set at $5 \%$ of the maximal stimulator output to minimize magnetic stimulation. Both groups were exposed to identical clicking sound generated during each session. As in the real rPMS group, the coil directly touched the skin of patients in the sham rPMS group, thereby exposing them to similar sensation [3]. The application of rPMS coil to both groups of patients was conducted by experienced physical therapists with sufficient preliminary training on the application of rPMS before the study. Physical therapists applying the magnetic stimulation could not be blinded. Thus, their conversion with patients was limited for both groups in order to minimize their effects on patients. Authors were blinded to group allocation of patients.

\section{Assessments}

For the evaluation of back pain, VAS was used as the primary endpoint. Oswestry Disability Index (ODI) and RMDQ were employed as secondary endpoints. ODI is a tool that can evaluate functional impairment of a low back pain patient. It has a value of $0 \%$ to $100 \%$, with higher value indicating higher level of disability. The validity of ODI has long been proven and its ability to dis- cern levels of functional disability has been extensively documented [19]. RMDQ evaluates limitations in activities of daily living due to low back pain with 24 questions, with higher number of positive answers indicating more severe functional disability. The reliability and validity of RMDQ have been confirmed [20]. Each assessment was conducted via subjects completing questionnaires with assistance from an independent researcher who was blinded as to group allocation. VAS was evaluated before and after every session whereas ODI and RMDQ were assessed at the baseline and after sessions 5 and 10 .

\section{Statistical analysis}

In normal distribution evaluation using KolmogorovSmirnov test and Shapiro-Wilk test, normality of either group was satisfied. Accordingly, statistical analysis was performed using the nonparametric method. MannWhitney U-test was used to compare the two groups for each session evaluated. Wilcoxon signed-rank test was employed to compare differences between pre-manipulation and post-manipulation. Courses of pain relief and functional recovery were analyzed by repeated-measures analysis of variance (ANOVA). Post-hoc analyses were undertaken using Bonferroni correction. Pearson correlation test was performed to assess changes in pain and disability. A p-value $<0.05$ was considered statistically significant. SPSS version 21.0 (IBM SPSS, Armonk, NY, USA) was used for all statistical analyses.

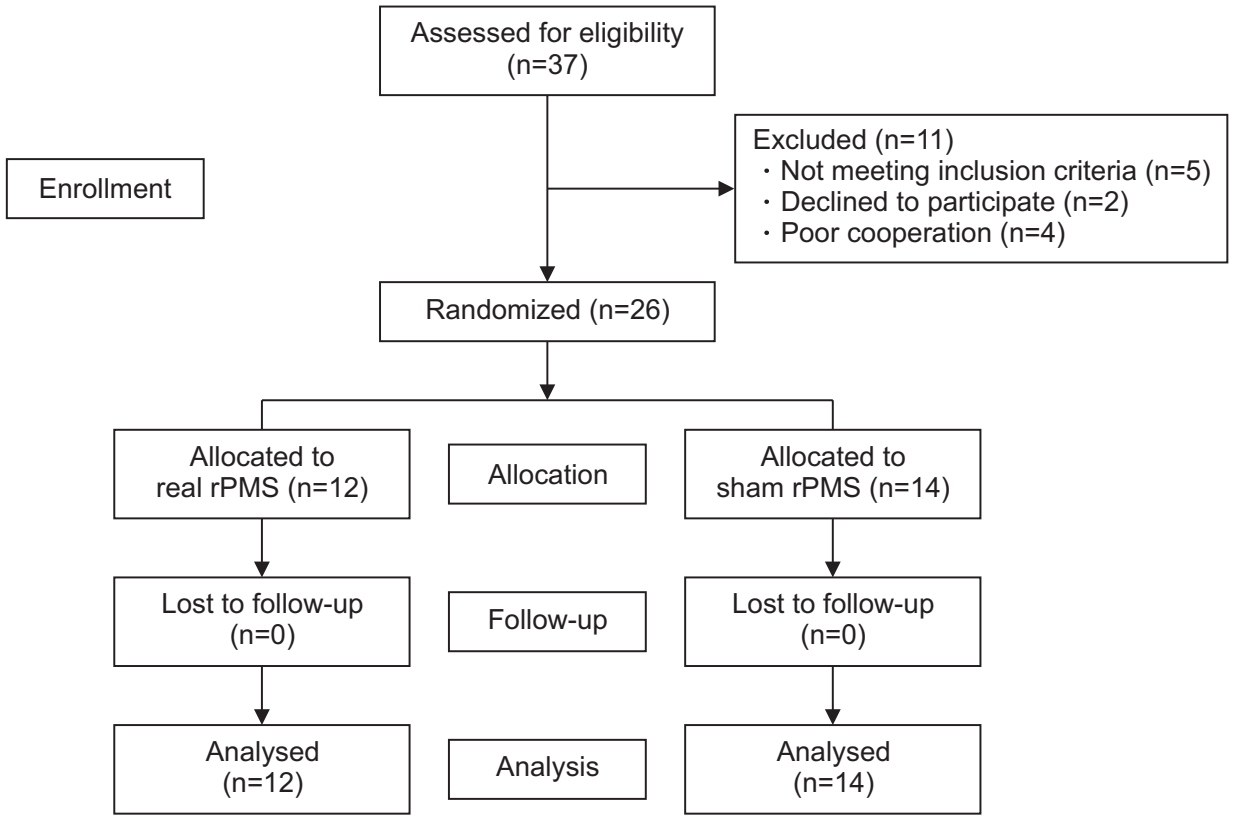

Fig. 1. Consort chart of the study design. rPMS, repetitive peripheral magnetic stimulation. 


\section{RESULTS}

\section{Baseline characteristics of subjects}

In the present study, a total of 26 ALBP patients were enrolled, including 18 patients diagnosed with lumbar sprain and 8 patients diagnosed with lumbar herniated nucleus pulposus. Lumbar herniated nucleus pulposus was identified through magnetic resonance imaging study. Only those patients without any motor weakness were included in this study. Twelve and 14 patients were randomly allocated to the real rPMS group and the sham rPMS group, respectively. All subjects completed a total of 10 treatment sessions (Fig. 1). Baseline characteristics of subjects in the two groups are summarized in Table 1. There were no significant differences in baseline characteristics between the two groups.

\section{Immediate effects of rPMS on ALBP}

According to VAS results, significant pain reduction was observed after each session compared to VAS evaluated before each session in the real rPMS group, with average VAS gap (VAS after session - VAS before session) of $-12.42 \pm 8.71$. By contrast, subjects in the sham rPMS group showed marginal changes, with average VAS gap of $-1.00 \pm 4.67$. There was no significant change in VAS gap in any session for this group (Fig. 2). Mann-Whitney U-test was used to compare VAS gaps of each session between the two groups. Pain relief in the real rPMS group was found to be significantly greater than in the sham rPMS group for all sessions except session 9 .

\section{Persistent effects of rPMS on ALBP}

Repeated-measures ANOVA showed significant VAS differences over the passage of time in both the real rPMS group and the sham rPMS group (baseline, after session

Table 1. Baseline characteristics of subjects

\begin{tabular}{lccc}
\hline & $\begin{array}{c}\text { Real rPMS } \\
\text { group } \\
(\mathbf{n}=\mathbf{1 2})\end{array}$ & $\begin{array}{c}\text { Sham rPMS } \\
\text { group } \\
(\mathbf{n}=\mathbf{1 4})\end{array}$ & p-value \\
\hline Age $(\mathrm{yr})$ & $52.50 \pm 21.45$ & $51.21 \pm 17.98$ & 0.869 \\
Sex $(\mathrm{male}:$ female $)$ & $6: 6$ & $6: 8$ & 0.512 \\
Height $(\mathrm{m})$ & $1.64 \pm 0.11$ & $1.62 \pm 0.10$ & 0.519 \\
Body weight $(\mathrm{kg})$ & $67.06 \pm 15.24$ & $63.31 \pm 11.64$ & 0.485 \\
BMI $\left(\mathrm{kg} / \mathrm{m}^{2}\right)$ & $24.5 \pm 4.23$ & $23.94 \pm 3.17$ & 0.705 \\
Diagnosis $(\mathrm{LS}:$ LHNP) & $8: 4$ & $10: 4$ & 0.563 \\
\hline VAS & $63.75 \pm 14.64$ & $66.07 \pm 12.28$ & 0.705 \\
ODI & $50.98 \pm 10.70$ & $51.87 \pm 8.86$ & 0.899 \\
\hline RMDQ & $13.67 \pm 3.82$ & $13.79 \pm 3.09$ & 0.860 \\
\hline
\end{tabular}

Values are presented as mean \pm standard deviation.

rPMS, repetitive peripheral magnetic stimulation; BMI, body mass index; LS, lumbar sprain; LHNP, lumbar herniated nucleus pulposus; VAS, visual analogue scale; RMDQ, Korean version of Roland-Morris Disability Questionnaire; ODI, Korean version of Oswestry Disability Index.
(A)

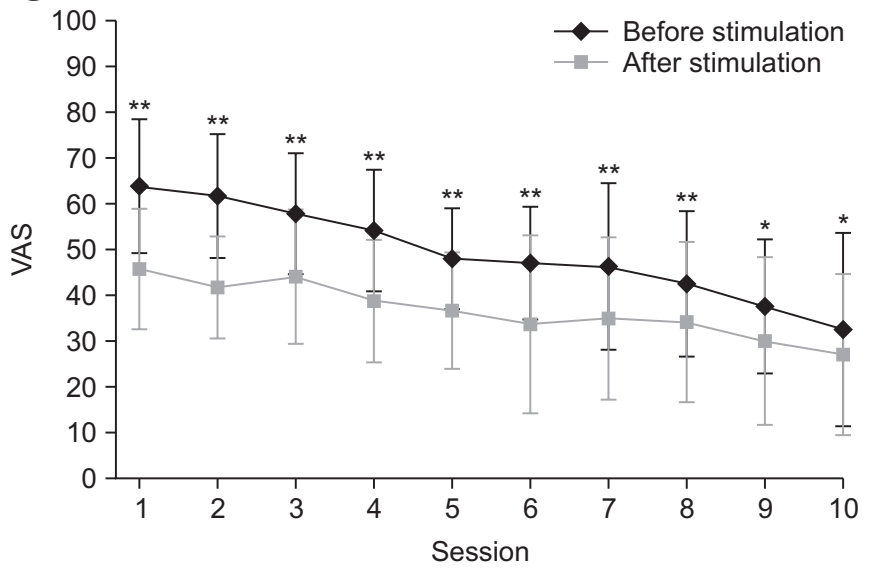

(B)

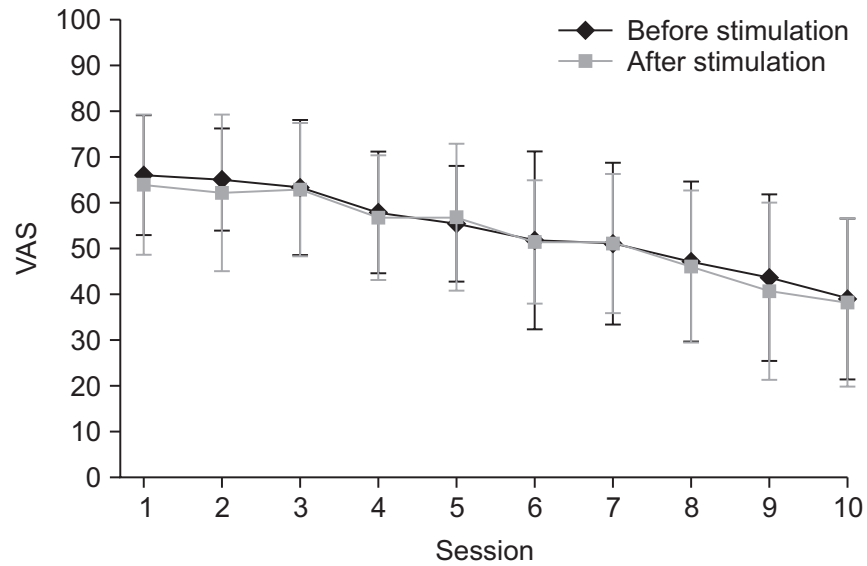

Fig. 2. Changes in VAS before and after each session observed in the real rPMS group (A) and the sham rPMS group (B). In the real rPMS group, VAS was reduced after every session compared to VAS measured before such session. The sham rPMS group showed no significant VAS change in any session. VAS, visual analogue scale; rPMS, repetitive peripheral magnetic stimulation. ${ }^{*} \mathrm{p}<0.05,{ }^{* *} \mathrm{p}<0.01$. 
5, and after session 10: $\left.\mathrm{F}_{(2,48)}=86.07, \mathrm{p}<0.001\right)$. There were also significant VAS differences between the two groups $\left(F_{(1,24)}=4.35, p=0.048\right)$. Difference in the change of VAS between the two groups over time was also significant (baseline, after session 5, and after session 10: $\left(\mathrm{F}_{(2,48)}=6.51\right.$, $\mathrm{p}=0.003$ ) (Fig. 3A). Bonferroni post-hoc tests showed that the VAS of the real rPMS group was significantly decreased both after session $5(36.67 \pm 12.67, \mathrm{p}<0.001)$ and after session $10(27.08 \pm 17.64, \mathrm{p}<0.001)$ compared to that at baseline (63.75 \pm 14.64$)$. On the other hand, the VAS of the sham rPMS group was only significantly decreased after session $10(38.21 \pm 18.25, \mathrm{p}<0.001)$. VAS was not significantly decreased after session $5(56.79 \pm 16.01, \mathrm{p}=0.377)$ compared to that at baseline $(66.07 \pm 12.28)$ in this group.

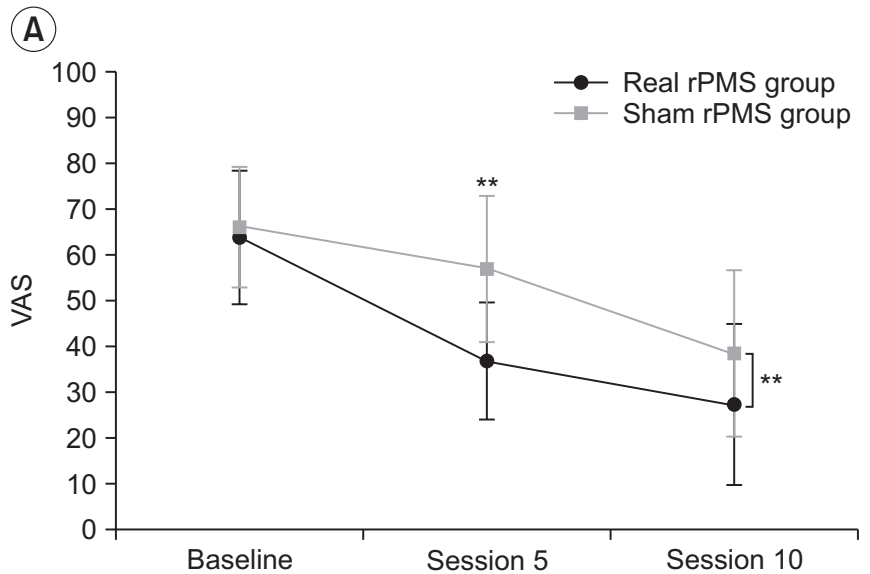

(C)

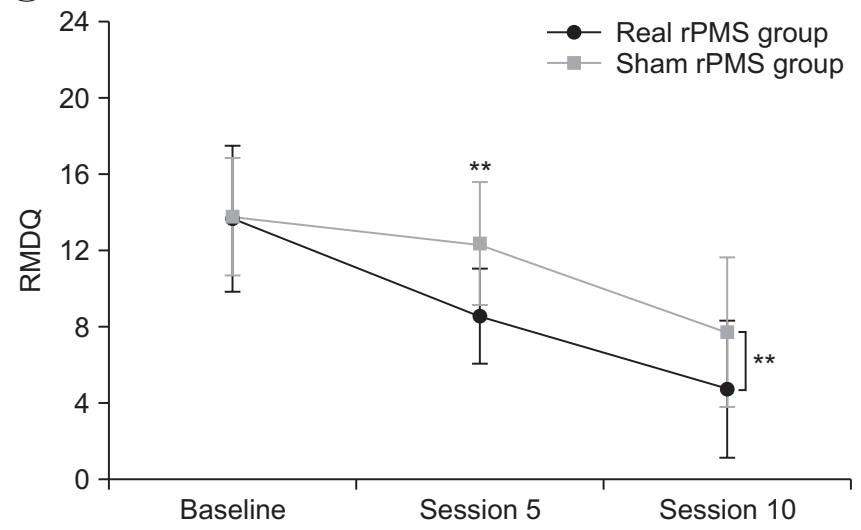

Mann-Whitney U-test was used to compare the VAS after each session between the two groups. Results showed that the real rPMS group demonstrated significant more pain relief compared to the sham rPMS group until session 7 . In sessions 8 to 10 , the VAS of the real rPMS group was lower than that of sham rPMS group by an average of 11.25 \pm 7.06 . However, the difference between the two groups was not statistically significant (Fig. 4A). We also compared baseline VAS and VAS before each session to confirm the persistence of pain relief under conditions that excluded the effect of immediate pain reduction (right after rPMS treatment). The real rPMS group showed significant improvement is VAS evaluated before session 2 and sessions thereafter compared to baseline

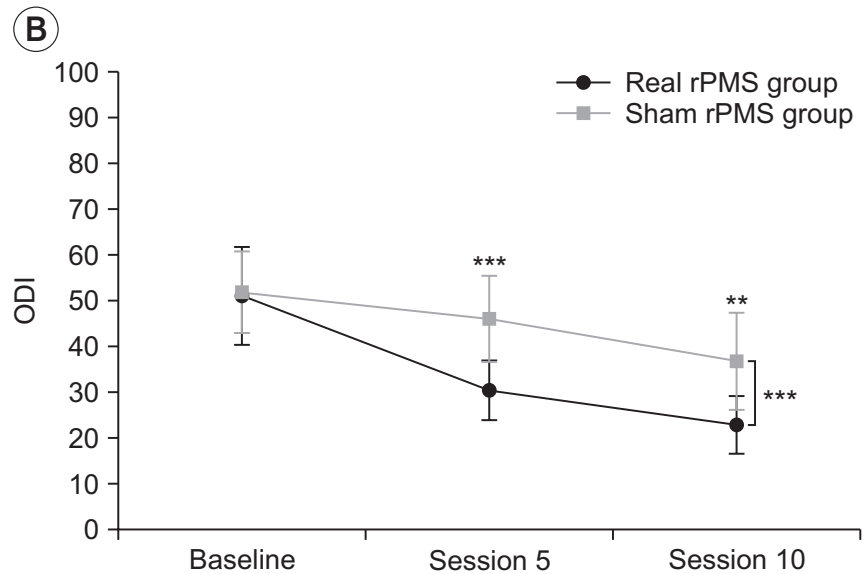

Fig. 3. Changes in VAS (A), ODI (B), and RMDQ (C) of the real rPMS group and sham rPMS group according to passage of time. Differences in changes of the VAS, ODI, and RMDQ over the course of time between the two groups were significant ( $\mathrm{p}=0.003, \mathrm{p}<0.001$, and $\mathrm{p}=0.006$, respectively). In comparing the two groups, there was a significant difference in VAS at session 5, but not at session 10; ODI showed a significant difference at sessions 5 and 10; and there was a significant difference in RMDQ at session 5 but not at session 10. VAS, visual analogue scale; ODI, Korean version of Oswestry Disability Index; RMDQ, Korean version of Roland-Morris Disability Questionnaire; rPMS, repetitive peripheral magnetic stimulation. ${ }^{*} \mathrm{p}<0.05,{ }^{* *} \mathrm{p}<0.01,{ }^{* * *} \mathrm{p}<0.001$. 

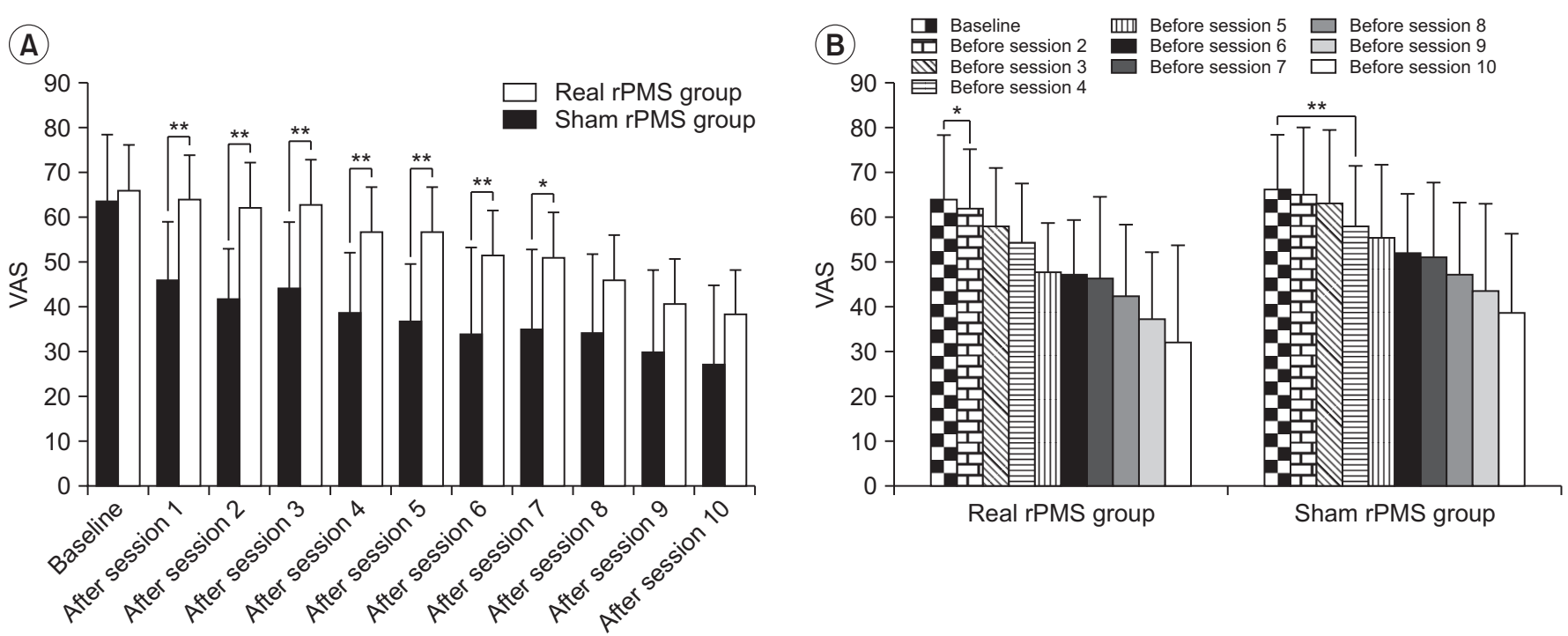

Fig. 4. Comparison of VAS between the real rPMS group and the sham rPMS group after each session. (A) From sessions 1 to 7, the VAS of the real rPMS group evaluated after each session was significantly lower than that of the sham rPMS group. Changes in VAS evaluated before each session of the real rPMS group and sham rPMS group. (B) Compared to baseline VAS, the real rPMS group showed significant VAS reduction starting from session $2(\mathrm{p}=0.025)$ whereas the sham rPMS group showed significant VAS reduction starting from session $4(\mathrm{p}=0.003)$. VAS, visual analogue scale; rPMS, repetitive peripheral magnetic stimulation. ${ }^{*} \mathrm{p}<0.05,{ }^{* *} \mathrm{p}<0.01$.

VAS (the first comparison), confirming 'sustained' pain relief effect of rPMS. On the other hand, the sham rPMS group showed significant improvement in VAS evaluated before session 4 and sessions thereafter compared to baseline VAS (Fig. 4B).

\section{Functional recovery}

Results of repeated-measures ANOVA showed significant differences in ODI $\left(\mathrm{F}_{(2,48)}=86.07, \mathrm{p}<0.001\right)$ and RMDQ $\left(F_{(2,48)}=86.07, p<0.001\right)$ over the passage of time (baseline, after session 5, and after session 10) in both the real rPMS group and the sham rPMS group. There was a significant difference in ODI between the two groups $\left(F_{(1,24)}=11.19, p=0.003\right)$. However, the difference in RMDQ was only marginally insignificant $\left(\mathrm{F}_{(1,24)}=3.83, \mathrm{p}=0.062\right)$. Difference in changes of ODI $\left(\mathrm{F}_{(2,48)}=12.84, \mathrm{p}<0.001\right)$ and $\operatorname{RMDQ}\left(\mathrm{F}_{(2,48)}=5.76, \mathrm{p}=0.006\right)$ over the course of time were significant between the two groups (Fig. 3B, 3C). Bonferroni post hoc tests showed that the ODI of the real rPMS group was significantly decreased both after session 5 $(30.39 \pm 6.53, \mathrm{p}<0.001)$ and after session $10(22.74 \pm 6.29$, $\mathrm{p}<0.001)$ compared to baseline ODI $(50.98 \pm 10.70)$. On the other hand, the ODI of the sham rPMS group was only significantly decreased after session $10(36.70 \pm 10.74$, $\mathrm{p}=0.001$ ) without showing significant decrease after ses- sion $5(45.97 \pm 9.45, \mathrm{p}=0.348)$ compared to baseline ODI (51.87 \pm 8.86$)$. The RMDQ of the real RPMS group was also significantly decreased both after session $5(8.58 \pm 2.50$, $\mathrm{p}=0.002)$ and session $10(4.75 \pm 3.60, \mathrm{p}<0.001)$ compared to baseline RMDQ (13.67 \pm 3.82$)$. The RMDQ of the sham rPMS group was only decreased significantly after session $10(7.71 \pm 3.93, \mathrm{p}<0.001)$ compared to baseline RMDQ $(13.79 \pm 3.09)$ without showing significant decrease after session 5 (12.36 $\pm 3.23, \mathrm{p}=0.834)$. Results of MannWhitney U-test showed that, after session $5(\mathrm{p}<0.001)$ and session $10(\mathrm{p}=0.002)$, the real $\mathrm{rPMS}$ group showed significant functional superiority compared to the sham rPMS group based on ODI (Fig. 3B). The real rPMS group also showed significant functional improvement in terms of RMDQ compare to the sham rPMS group when they were evaluated after session $5(\mathrm{p}=0.004)$. However, there was no significant difference in functional improvement between the two groups after session $10(\mathrm{p}=0.095)$ (Fig. 3C). Pearson correlation coefficient analysis indicated that decrease of VAS in the real rPMS group was strongly correlated with decrease of ODI $(\mathrm{r}=0.680, \mathrm{p}=0.015)$ and RMDQ ( $r=0.934, p<0.001)$. Pearson correlation coefficient analysis also showed that that the decrease of VAS in the sham rPMS group was significantly correlated with decrease of ODI $(r=0.650, p=0.012)$ and RMDQ $(r=0.879$, 
$\mathrm{p}<0.001)$

\section{DISCUSSION}

The purpose of this study was to determine the effects of real rPMS on pain relief and functional recovery in ALBP patients compared to sham rPMS. As we postulated, real rPMS showed effects of immediate pain reduction and sustained pain relief, leading to early functional recovery. In three of four studies conducted previously, effects of immediate pain relief through rPMS were confirmed in patients with CLBP [13-15]. The remaining one was evaluated 8 hours after rPMS was administered. Accordingly, it was difficult to assess the effects accurately [12]. In this study on ALBP patients, immediate and significant pain improvement was also confirmed after each session in the real rPMS group. The mechanism of such effects has not yet been sufficiently studied or discussed. One possible explanation is based on the gate control theory. The electrical field formed by magnetic stimulation dominantly might depolarize large diameter myelinated $A \beta$ afferent fibers due to their high conduction velocity, thereby inhibiting the depolarization of relatively small diameter $\mathrm{A} \delta$ nerve fibers and $\mathrm{C}$ nerve fibers which in turn can block pain signals from traveling to the brain [21]. However, the gate control theory alone cannot fully explain the immediate effects in relieving the pain by magnetic stimulation given that magnetic stimulation is relatively ineffective in recruiting cutaneous sensory afferents. This is because magnetic stimulation bypasses the cutaneous layer with minimal resistance to penetrate to deeper layers such as muscles and spinal roots. It directly recruits proprioceptive afferents (types Ia, Ib, II). Another possibility involves the immediate activation of a descending inhibitory pathway. However, there is no evidence or study supporting that rPMS activates the brain stem area such as rostral ventral medulla (RVM) and periaqueductal gray (PAG) that constitute this pathway. Furthermore, intensities of magnetic stimulation applied in this study were not robust enough to generate nociceptive signals.

The immediate effect of pain reduction of rPMS is not limited to low back pain patients. Previous studies have shown significant pain relief right after application of rPMS in various types of musculoskeletal pain including myofascial pain syndrome. This suggests that rPMS can initiate immediate pain modulatory mechanisms in general. However, no clear explanation has been provided yet. Accordingly, further research on this subject is warranted.

Through this study, the persistence of pain relief since session 1 in the real rPMS group was observed. To confirm whether the effect from the first session persisted, we compared VAS before the second session to baseline VAS and observed that the real rPMS group showed significant improvement in pain relief. Following each session, the VAS before such session was compared to baseline VAS, through which we confirmed that there was significant improvement. However, in the sham rPMS group, we began to see significant improvement only starting from session 4. As reviewed in the introduction section, most cases of ALBP show rapid improvement within weeks. Therefore, the improvement observed in the sham rPMS group can be attributable to the natural course of the disease. These effects are consistent with those found in studies conducted on CLBP patients. They have been described as 'residual,' 'persistent', 'maintained, or 'long-term' relief of pain [13-15]. The most likely explanation for this is improvement in motor control by rPMSinduced brain plasticity.

CLBP patients typically undergo changes in motor coordination of abdominal and paravertebral muscles, resulting in decrease in spine control. Persistence of low back pain may be due to microtrauma caused by motor control impairment of these adjacent trunk muscles supporting the spine [22-24]. There is an increasing amount of evidence suggesting that such alterations in motor control are caused by plastic changes in the sensorimotor cortex that is responsible for planning movements [25]. For example, changes in M1 excitability have been observed in CLBP patients through motor evoked potential (MEP) studies [26,27]. Substantial reorganization of the primary somatosensory cortex (S1) and impairment of connectivity with the M1 cortex have been confirmed by magnetic source imaging [28].

Once rPMS activates muscles, proprioceptive afferents are generated through two pathways: indirect activation of mechanoreceptors on fibers (type Ia, Ib, II), and direct activation of sensorimotor nerve fibers [29]. This proprioceptive influx into the brain is believed to cause cortical plasticity [25,30-32]. Studies using MEP recruitment curves have confirmed that corticospinal excitability is 
maintained for up to 60 minutes if rPMS is conducted at a frequency of $25 \mathrm{~Hz}$ [31]. The duration of corticospinal excitability cannot be 60 minutes in all cases since there may be differences in the duration of cortical activation depending on the frequency of the rPMS applied. However, the fact that pain relief persisted long after the excited period suggests the possibility of long-term potentiation due to brain plasticity. In fact, activation of the premotor cortex and the posterior parietal cortex, both of which are closely related to motor control, has been confirmed on functional magnetic resonance imaging (fMRI) after rPMS treatment [30]. Improvement in the motor control of subjects is also confirmed [30]. In addition, it has been confirmed that the real rPMS group shows more distinct difference in activation of the precentral area (M1) and the postcentral area (S1) compared to the sham rPMS group through fMRI [31]. Increased excitability of these sensorimotor cortexes involved in motor planning and control may potentiate motor control improvement. This could lead to efficient protection of the spine from microtraumas in daily activities, thus enabling persistence of pain reduction. However, this effect is known to be further enhanced when rPMS is combined with motor training $[14,15,25]$. One limitation of this study was that we did not create a group of ALBP patients who carried out lumbar exercise.

In this study involving ALBP patients as in the rPMS study of CLBP patients, the pain relief effect did not remain transient. Based on knowledge gained from past researches, we postulate that this is because magnetic stimulation might have activated a modulatory mechanism which maintains pain relief at brain level in ALBP patients. Although ALBP patients are less extensively documented than CLBP patients, they also show changes in trunk muscle activities. One study with experimentally induced acute back pain patients has suggested that changes in immediate motor control are caused by acute pain. Although there is variability depending on muscles, electromyographic studies and researches using MEP have shown that muscles such as abdominal external oblique and lumbar erector spinae in the superficial layer are activated in experimentally-induced acute back pain patients. This might have the effect of splinting the trunk to protect further injury. As a result, the mobility of the spine is reduced. On the other hand, decreased activities and delayed recruitment have been observed for transversus abdominis located relatively in a deeper layer $[33,34]$. This may reduce the stability of the spine itself. As a result, physiologic motion of the spine does not occur in daily life. Once the stability of the spine is reduced, it may become vulnerable to microtraumas. This might interfere with pain relief in patients with ALBP. In a previous MEP study on induced acute back pain subjects, the difference in cortical excitability according to the type of trunk muscles [34] suggests possible occurrence of corticomotor plasticity in ALBP patients as in CLBP patients. In addition, the neuromodulatory effect of an rPMS therapy might be achieved at the brain level in ALBP patients. However, advanced studies addressing topics such as which area of the brain undergo changes in ALBP patients are lacking. Further study is needed to obtain a more convincing explanation. In this study, the persistence of pain relief due to real rPMS treatment was confirmed. It is noteworthy that significant pain relief can be expected in patients with ALBP who receive rPMS treatment compared to patients who do not, at least for the first 9 days (i.e., 7 sessions).

Another important effect of rPMS identified in this study is early functional recovery. It seems that improvement in disability can be attributable to decrease of pain in the first place. Correlation coefficient analysis showed a significantly positive correlation of the VAS with ODI and RMDQ. In addition, deducing from studies conducted on CLBP patients, improvement in motor control might have contributed to functional recovery. According to studies performed so far, differential muscle activation identified in induced acute back pain subjects seems to be related to changes at brain level that might inhibit functional movement of the spine [33,34]. As with CLBP patients, if rPMS is shown to reverse brain plasticity caused by acute pain, we might be able to provide a clear explanation on functional recovery based on rPMS treatments. To this end, further study is needed to determine whether motor control is improved and how the sensorimotor cortex is reorganized when rPMS is applied to ALBP patients.

In conclusion, rPMS treatment appears to have effects of immediate pain reduction as well as sustained pain relief. It can also induce early functional recovery for patients with ALBP. Therefore, rPMS treatment could be used as an effective option for patients with ALBP seeking medical care. 


\section{CONFLICT OF INTEREST}

No potential conflict of interest relevant to this article was reported.

\section{REFERENCES}

1. Murray CJ, Vos T, Lozano R, Naghavi M, Flaxman AD, Michaud C, et al. Disability-adjusted life years (DALYs) for 291 diseases and injuries in 21 regions, 1990-2010: a systematic analysis for the Global Burden of Disease Study 2010. Lancet 2012;380:2197-223.

2. van Tulder M, Becker A, Bekkering T, Breen A, del Real MT, Hutchinson A, et al. Chapter 3. European guidelines for the management of acute nonspecific low back pain in primary care. Eur Spine J 2006;15 Suppl 2:S169-91.

3. Henschke N, Maher CG, Refshauge KM, Herbert RD, Cumming RG, Bleasel J, et al. Prognosis in patients with recent onset low back pain in Australian primary care: inception cohort study. BMJ 2008;337:a171.

4. Downie AS, Hancock MJ, Rzewuska M, Williams CM, Lin CW, Maher CG. Trajectories of acute low back pain: a latent class growth analysis. Pain 2016;157:22534.

5. Dahm KT, Brurberg KG, Jamtvedt G, Hagen KB. Advice to rest in bed versus advice to stay active for acute low-back pain and sciatica. Cochrane Database Syst Rev 2010;(6):CD007612.

6. Saragiotto BT, Machado GC, Ferreira ML, Pinheiro MB, Abdel Shaheed C, Maher CG. Paracetamol for low back pain. Cochrane Database Syst Rev 2016;(6): CD012230.

7. Harirforoosh S, Asghar W, Jamali F. Adverse effects of nonsteroidal antiinflammatory drugs: an update of gastrointestinal, cardiovascular and renal complications. J Pharm Pharm Sci 2013;16:821-47.

8. Kinkade S. Evaluation and treatment of acute low back pain. Am Fam Physician 2007;75:1181-8.

9. Acute Low Back Problems Guideline Panel. Acute low back problems in adults: assessment and treatment. Am Fam Physician 1995;51:469-84.

10. Furlan AD, Giraldo M, Baskwill A, Irvin E, Imamura M. Massage for low-back pain. Cochrane Database Syst Rev 2015;(9):CD001929.

11. Beaulieu LD, Schneider C. Repetitive peripheral magnetic stimulation to reduce pain or improve sensorimotor impairments: a literature review on parameters of application and afferents recruitment. Neurophysiol Clin 2015;45:223-37.

12. Kim JY, Yoon SH, Rah UW, Cho KH, Hong JY. Effect of repetitive magnetic stimulation and transcutaneous electrical nerve stimulation in chronic low back pain: a pilot study. J Korean Acad Rehabil Med 2010;34:7259.

13. Lo YL, Fook-Chong S, Huerto AP, George JM. A randomized, placebo-controlled trial of repetitive spinal magnetic stimulation in lumbosacral spondylotic pain. Pain Med 2011;12:1041-5.

14. Masse-Alarie H, Flamand VH, Moffet H, Schneider C. Peripheral neurostimulation and specific motor training of deep abdominal muscles improve posturomotor control in chronic low back pain. Clin J Pain 2013; 29:814-23.

15. Masse-Alarie H, Beaulieu LD, Preuss R, Schneider C. Repetitive peripheral magnetic neurostimulation of multifidus muscles combined with motor training influences spine motor control and chronic low back pain. Clin Neurophysiol 2017;128:442-53.

16. Maccabee PJ, Amassian VE, Cracco RQ, Cadwell JA. An analysis of peripheral motor nerve stimulation in humans using the magnetic coil. Electroencephalogr Clin Neurophysiol 1988;70:524-33.

17. Smania N, Corato E, Fiaschi A, Pietropoli P, Aglioti SM, Tinazzi M. Therapeutic effects of peripheral repetitive magnetic stimulation on myofascial pain syndrome. Clin Neurophysiol 2003;114:350-8.

18. Smania N, Corato E, Fiaschi A, Pietropoli P, Aglioti SM, Tinazzi M. Repetitive magnetic stimulation: a novel therapeutic approach for myofascial pain syndrome. J Neurol 2005;252:307-14.

19. Saltychev M, Mattie R, McCormick Z, Barlund E, Laimi K. Psychometric properties of the Oswestry Disability Index. Int J Rehabil Res 2017;40:202-8.

20. Stevens ML, Lin CC, Maher CG. The Roland Morris Disability Questionnaire. J Physiother 2016;62:116.

21. Melzack R, Wall PD. Pain mechanisms: a new theory. Science 1965;150:971-9.

22. Massion J. Movement, posture and equilibrium: interaction and coordination. Prog Neurobiol 1992;38:3556.

23. Tsao H, Danneels LA, Hodges PW. ISSLS prize winner: 
smudging the motor brain in young adults with recurrent low back pain. Spine (Phila Pa 1976) 2011;36: 1721-7.

24. Tsao H, Danneels L, Hodges PW. Individual fascicles of the paraspinal muscles are activated by discrete cortical networks in humans. Clin Neurophysiol 2011; 122:1580-7.

25. Masse-Alarie H, Schneider C. Revisiting the corticomotor plasticity in low back pain: challenges and perspectives. Healthcare (Basel) 2016;4:67.

26. Masse-Alarie H, Beaulieu LD, Preuss R, Schneider C. The side of chronic low back pain matters: evidence from the primary motor cortex excitability and the postural adjustments of multifidi muscles. Exp Brain Res 2017;235:647-59.

27. Strutton PH, Theodorou S, Catley M, McGregor AH, Davey NJ. Corticospinal excitability in patients with chronic low back pain. J Spinal Disord Tech 2005;18: 420-4.

28. Flor H, Braun C, Elbert T, Birbaumer N. Extensive reorganization of primary somatosensory cortex in chronic back pain patients. Neurosci Lett 1997;224:58.

29. Struppler A, Angerer B, Gundisch C, Havel P. Modula- tory effect of repetitive peripheral magnetic stimulation on skeletal muscle tone in healthy subjects: stabilization of the elbow joint. Exp Brain Res 2004;157:5966.

30. Struppler A, Binkofski F, Angerer B, Bernhardt M, Spiegel S, Drzezga A, et al. a fronto-parietal network is mediating improvement of motor function related to repetitive peripheral magnetic stimulation: a PETH2O15 study. Neuroimage 2007;36 Suppl 2:T174-86.

31. Gallasch E, Christova M, Kunz A, Rafolt D, Golaszewski S. Modulation of sensorimotor cortex by repetitive peripheral magnetic stimulation. Front Hum Neurosci 2015;9:407.

32. Krause P, Straube A. Peripheral repetitive magnetic stimulation induces intracortical inhibition in healthy subjects. Neurol Res 2008;30:690-4.

33. Hodges PW, Moseley GL, Gabrielsson A, Gandevia SC. Experimental muscle pain changes feedforward postural responses of the trunk muscles. Exp Brain Res 2003;151:262-71.

34. Tsao H, Tucker KJ, Hodges PW. Changes in excitability of corticomotor inputs to the trunk muscles during experimentally-induced acute low back pain. Neuroscience 2011;181:127-33. 